\title{
HABILIDADES MATEMÁTICAS INICIALES Y DIFICULTADES MATEMÁTICAS PERSISTENTES
}

\author{
Rebeca Siegenthaler Hierro \\ Universidad Jaume I de Castellón \\ (siegenth@uji.es) \\ Ana Miranda Casas \\ Universidad de Valencia \\ Jessica Mercader Ruiz \\ Universidad Jaume I de Castellón \\ Ma Jesús Presentación Herrero \\ Universidad Jaume I de Castellón \\ https://doi.org/10.17060/ijodaep.2017.n1.v3.992
}

Fecha de Recepción: 9 Febrero 2017

Fecha de Admisión: 1 Abril 2017

\section{RESUMEN}

El objetivo del presente estudio consistió en examinar las diferencias en determinadas competencias matemáticas básicas (e.g. conteo, operaciones lógicas, habilidades de comparación de magnitudes) evaluadas en Educación Infantil (EI) entre sujetos con dificultades persistentes en el área de matemáticas en $2^{0}$ de Educación Primaria (EP) y niños con un rendimiento adecuado. La muestra final del estudio estuvo conformada por 180 niños (inicialmente, compuesta por 209 preescolares), de los cuales un $6.6 \%$ mostraron dificultades matemáticas persistentes ( $n=12)$ y el $45.5 \%$ presentaron un rendimiento adecuado en ambas mediciones $(n=82)$. En El, se administraron las tareas de conteo (procedimental y conceptual), operaciones lógicas (seriación, clasificación, conservación e inclusión numérica) y comparación de magnitudes (simbólica y no-simbólica) de la prueba TEDI-MATH (Grégoire, Noël, y Van Nieuwenhoven, 2005). Los resultados evidenciaron diferencias en 4 de las 8 competencias matemáticas básicas analizadas en El, con efectos especialmente importantes en las habilidades para comparar números arábigos (comparación simbólica) y para manejar la secuencia numérica verbal (conteo procedimental). Se comentan las implicaciones de estos hallazgos para la investigación y la práctica psicoeducativa.

Palabras clave: dificultades matemáticas, estudio retrospectivo, competencias matemáticas básicas, persistencia.

\section{ABSTRACT}

Initial math skills and persistent math difficulties

The aim of the present study was to analyse the differences in certain basic mathematical competences (for example, counting, logical operations, magnitude comparability skills) assessed in 


\section{HABILIDADES MATEMÁTICAS INICIALES Y DIFICULTADES MATEMÁTICAS PERSISTENTES}

Early Childhood Education between children with persistent difficulties in the area of mathematics in 2nd Primary Education and children with adequate performance. The final sample of the study consisted of 180 children (composed initially of 209 pre-schoolers), of which $6.6 \%$ showed persistent mathematics difficulties $(n=12)$ and $45.5 \%$ presented an adequate performance in both measurements $(n=82)$. In Early Childhood Education we administered counting, logical operations (seriation, classification, conservation and numerical inclusion) and comparison of magnitudes (symbolic and non-symbolic) of the TEDI-MATH test (Grégoire, Noël, \& Van Nieuwenhoven, 2005). Results evidenced differences in 4 of the 8 basic mathematical competences analysed in Early Childhood Education, with especially significant effects on the ability to compare symbolic numbers and to manage verbal numerical sequence. The implications of these findings for psychoeducational research and practice are discussed.

Key words: mathematical difficulties, retrospective study, basic mathematical competences, persistence.

\section{ANTECEDENTES DE LA TEMÁTICA A TRATAR}

Los informes internacionales ponen de manifiesto la existencia de un elevado porcentaje de sujetos que manifiestan dificultades ante tareas básicas de matemáticas (Mullis, Martin, Foy, y Hooper, 2016). Del mismo modo, se refleja que el logro global que alcanzan los estudiantes de nuestro país en esta disciplina es especialmente bajo, con un porcentaje de alumnos que presentan dificultades para la realización de tareas matemáticas sencillas del 26\%. Las dificultades matemáticas pueden tener consecuencias negativas importantes en la vida de las personas ya que las habilidades matemáticas son básicas para muchos aspectos de la vida y el trabajo. Actualmente, no existe un consenso sobre los factores explicativos del rendimiento matemático. Este hecho, unido a las consecuencias negativas que se asocian a éstas en distintas áreas del desarrollo evolutivo (Mazzocco y Räsänen, 2013), resalta la importancia de identificar los factores implicados en su desarrollo, con el fin de comprender su origen e intervenir temprana y preventivamente y con garantías de éxito (Geary, 2011).

Los investigadores en el campo de la educación y la psicología establecen que algunas competencias matemáticas básicas, como el conteo, las operaciones lógicas y la comparación de magnitudes, son especialmente necesarias para el aprendizaje matemático. Las habilidades matemáticas básicas en los primeros años pueden predecir el rendimiento matemático al finalizar la escolaridad (Morgan, Farkas y Wu, 2009), siendo éste un predictor de la empleabilidad y del salario en la adultez (Bynner, 2004). Los niños con dificultades en matemáticas muestran déficits en estas habilidades (Geary, Hoard, Nugent, y Bailey, 2013).

Diferentes estudios avalan el poder predictivo del conteo temprano (entre otras competencias básicas) sobre el rendimiento matemático en El y en etapas iniciales de la EP (Aunio y Niemivirta, 2010; Östergren y Träff, 2013). Además, Ios sujetos con dificultades específicas en matemáticas presentan problemas procedimentales en el conteo, realizando más errores y/0 utilizando estrategias inmaduras durante más tiempo (Geary y Hoard, 2005; Jordan y Montani, 1997; Ostad, 2000). Geary (2004) expone que dichos problemas procedimentales podrían explicarse en base a déficits en el conocimiento de los principios conceptuales necesarios para el conteo. Desde una perspectiva longitudinal, algunos trabajos muestran que el conteo (y otras competencias matemáticas básicas) en El juega un papel muy importante en la discriminación de niños con y sin dificultades matemáticas en el primer ciclo de EP (Stock, Desoete, y Roeyers, 2009a; Stock, Desoete, y Roeyers, 2009b; Stock, Desoete, y Roeyers, 2010).

En relación con las habilidades lógicas iniciales, diferentes trabajos relacionan el rendimiento matemático en los primeros cursos de EP con las competencias de seriación (Aunio y Niemivirta, 
2010; Tobia, Bonifacci, y Marzocchi, 2016) y clasificación (Stock et al., 2009a). Incluso, algunos autores resaltan el peso que estas habilidades básicas tienen en el rendimiento matemático a lo largo de toda la escolaridad (Desoete, Stock, Schapens, Baeyens, y Roeyers, 2009). La importancia de las operaciones lógicas tempranas se ha evidenciado no sólo para predecir el rendimiento posterior, sino también para determinar las dificultades futuras en esa área. Así, Stock et al. (2009a) informan del poder de las habilidades iniciales de seriación, clasificación y conservación (entre otras competencias matemáticas básicas) para discriminar entre sujetos con y sin riesgo de experimentar dificultades matemáticas en etapas posteriores. En esta línea, Stock et al. (2010) en una investigación longitudinal con niños de entre 5 y 8 años encontraron diferencias en seriación (evaluadas en El) entre niños con dificultades o bajo rendimiento persistente en matemáticas y niños con un rendimiento adecuado. Así, trabajos de intervención sugieren que los niños entrenados en razonamiento lógico tienen un progreso aritmético mayor que los niños no entrenados (Ping y GoldinMeadow, 2008).

La comparación de magnitudes, también parece ser una habilidad básica que se relaciona con el rendimiento matemático (Butterworth, 2005; De Smedt y Gilmore, 2011; Schleifer y Landerl, 2011). Sin embargo, los resultados sobre el peso relativo de esta habilidad así como su importancia en las dificultades matemáticas tempranas y persistentes no están claros (De Smedt, Noël, Gilmore, y Ansari, 2013). En cuanto a las habilidades no-simbólicas, un estudio realizado con niños de entre 3 y 5 años, Bonny y Lourenco (2013) encontraron una relación más fuerte entre habilidades de comparación no-simbólica y la competencia matemática en los niños con rendimiento más bajo. En este sentido, Stock et al. (2010) señalan que las habilidades de comparación no-simbólica son especialmente importantes para la discriminación entre sujetos con dificultades matemáticas persistentes y niños con bajo rendimiento matemático. Respecto a las habilidades simbólicas, Desoete, Ceulemans, De Weerdt y Pieters (2012) muestran que las deficiencias en estas habilidades discriminan a niños con dificultades matemáticas tanto en El, como en 2ํㅜ curso de EP. Además, en este mismo estudio, los déficits en tareas no-simbólicas que presentaban los sujetos con dificultades aritméticas en El no se mantuvieron en $2^{\circ}$ de EP. Los autores concluyen que una combinación de déficits en las habilidades tempranas de comparación simbólica y no-simbólica contribuye al riesgo de experimentar dificultades de aprendizaje posteriores en el área de matemáticas.

La revisión de la literatura en relación con la capacidad discriminativa de las habilidades matemáticas básicas entre niños con y sin dificultades persistentes de matemáticas, pone de manifiesto la necesidad de continuar investigando con el objetivo de clarificar el papel de las mismas en el rendimiento posterior. Son necesarios más trabajos longitudinales que identifiquen el peso de estas habilidades básicas con el fin de prevenir dificultades futuras a través de intervenciones específicas para ello.

\section{OBJETIVO DE LA INVESTIGACIÓN}

El objetivo de esta investigación es analizar las diferencias en las competencias matemáticas básicas evaluadas en El entre niños con dificultades persistentes y niños con rendimiento adecuado en el área de matemáticas dos años después, en $2^{\circ}$ de EP. Concretamente, las habilidades básicas que se analizan son conteo, operaciones lógicas y comparación de magnitudes.

\section{MUESTRA Y PARTICIPANTES}

En este trabajo han participado 180 niños escolarizados en diferentes centros educativos de las provincias de Castellón y Valencia y sus maestros. En la primera evaluación (T1) participaron 209 alumnos (52.2\% niños; $47.8 \%$ niñas) de 5 y 6 años (Media $=70.17$ meses; DT $=3.51$ meses), sus padres y maestros. Los niños cursaban en ese momento El. Ninguno de los participantes presenta- 


\section{HABILIDADES MATEMÁTICAS INICIALES Y DIFICULTADES MATEMÁTICAS PERSISTENTES}

ba discapacidad intelectual (Media $=98.63$; DT $=12.23$ ), deficiencias sensoriales graves, anomalías neurobiológicas, trastornos psicológicos o deprivación socio-cultural. El 88\% de los participantes tenía nacionalidad española, siendo el resto provenientes de otros países. Todos los sujetos hablaban y comprendían el español, independientemente de su nacionalidad. El $63.6 \%$ de niños asistían a centros públicos y el $36.4 \%$ a colegios concertados.

En la segunda evaluación (T2), dos años después, la muestra inicial se redujo a 180 alumnos (86.6\% de la misma) y sus maestros, debido a la mortandad experimental propia de los estudios longitudinales. En esta evaluación, los sujetos tenían entre 7 y 8 años (Media = 94.14 meses; DT = 3.61 meses), siendo el $51.1 \%$ niños y el $48.9 \%$ niñas. En el T2 los niños estaban cursando $2^{\circ}$ de EP en centros públicos (65\%) y concertados (35\%). En este momento, el $19.1 \%$ de los participantes asistía a sesiones con especialistas en los respectivos centros escolares en el T2: Apoyo Educativo (7.7\%), Educación Compensatoria (1.9\%), Pedagogía Terapéutica (3.3\%), Audición y Lenguaje $(3.8 \%)$ y tratamiento combinado $(2.4 \%)$.

La muestra fue dividida en función de la presencia de dificultades matemáticas a lo largo del tiempo. En el T1, siguiendo las directrices de Stock, et al. (2009a), se utilizaron los subtest de operaciones (con apoyo de imágenes, con enunciado aritmético y con enunciado verbal) de la prueba TEDI-MATH (Grégoire, et al., 2005) como indicadores de la competencia matemática. En el T2, se administró el test TEMA-3 (Gingsburg y Baroody, 2003) para estimar el rendimiento matemático. Un $6.6 \%$ de los sujetos $(n=12)$ mostraron dificultades persistentes en matemáticas, tomando como referencia los siguientes criterios: a) 1.5 desviaciones típicas por debajo de la media en los subtest de operaciones del TEDI-MATH en el T1; b) Un rendimiento inferior al PC 10 en la batería TEMA-3 en el T2. Como grupo de comparación, se seleccionaron aquellos sujetos que presentaron un rendimiento matemático igual o superior a la media en ambas mediciones $(n=82)$. Se encontraron diferencias estadísticamente significativas en la variable $\mathrm{Cl}\left(\operatorname{tg}_{92}=4.37 ; p<.001\right)$, que fueron tenidas en cuenta en los posteriores análisis.

\section{METODOLOGÍA}

\section{Instrumentos de evaluación}

Competencias matemáticas básicas (T1)

Para realizar esta investigación se aplicó el test de las Competencias Básicas en Matemáticas TEDI-MATH (Grégoire, et al., 2005), que es una prueba estandarizada para niños de entre 4 y 8 años de edad que evalúa las habilidades matemáticas básicas. Este test se agrupa en 6 subtest, que a su vez se desglosa en 25 pruebas. Las tareas seleccionadas para este trabajo fueron: operaciones lógicas (seriación, clasificación, conservación e inclusión) el conteo procedimental y conceptual (contar y numerar) y la comparación de magnitudes simbólica (comparación de números arábigos) y nosimbólica (estimación del tamaño) para evaluar las competencias matemáticas básicas de los participantes. La baremación española del instrumento cuenta con elevados índices de fiablidad y validez (Grégoire, et al., 2005)

\section{Rendimiento matemático (T2)}

Para realizar la clasificación de los niños en función de su rendimiento como bajo y medio, se aplicó el Test de Competencia Matemática Básica (TEMA-3; Gingsburg y Baroody, 2003). Se trata de una prueba estandarizada dirigida a niños entre 3 años y 8 años y 11 meses. Se compone de 72 ítems que valoran diferentes aspectos de la competencia matemática infantil. Contempla tanto aspectos informales (aquellos que no requieren el uso de símbolos matemáticos escritos), que son 
evaluados mediante 41 ítems, como aspectos formales (actividades que implican el uso de símboIos matemáticos), que se engloban en 31 ítems. Esta prueba cuenta con índices elevados de fiabilidad y validez en su baremación española (Gingsburg y Baroody, 2003). Para el presente estudio, se utilizan las puntuaciones directas en cada una de las subescalas y la puntuación total. Para el segundo objetivo, los participantes se clasifican en función del ICM.

\section{Procedimiento}

Para comenzar la investigación se obtuvieron los permisos de la Consellería de Educación de la Generalitat Valenciana, la aprobación por parte del Comité Ético de la Universitat Jaume I, así como el consentimiento informado de los padres. De los diferentes centros a los que se acudió, se seleccionaron 6 estudiantes por aula al azar mediante la técnica de muestreo aleatorio simple. La administración de las pruebas se produjo en horario lectivo sin interferir con las actividades significativas del currículum, en espacios cedidos por los centros escolares que reunían las condiciones de ventilación, aislamiento e iluminación óptimas para la evaluación psicopedagógica.

En el T1, se aplicaron las subpruebas seleccionadas del TEDI-MATH (Grégoire, et al., 2005), en una sesión de 30 minutos de duración aproximada. Dos cursos escolares después, se volvió a los colegios donde se administró la prueba estandarizada TEMA-3 (Gingsburg y Baroody, 2003) a los mismos sujetos, de forma individual y en una sesión de 30 minutos.

\section{Análisis estadísticos}

Los análisis estadísticos se realizaron con el software Statistical Package for the Social Science (SPSS), versión 22.00 (SPSS Inc., Chicago, IL USA). Se realizó un análisis multivariados de la covarianza (MANCOVA), introduciendo como co-variable el $\mathrm{Cl}$. Se introdujeron como variables dependientes las puntuaciones directas de ensayos correctos en las subpruebas de contar (conteo procedimental), numerar (conteo conceptual), operaciones lógicas (seriación, clasificación, conservación e inclusión), Comparación de números arábigos (comparación simbólica) y estimación de cantidades (comparación no-simbólica) del test TEDI-MATH (Grégoire, et al., 2005). Los efectos principales fueron comprobados $(p<.05)$, y se calculó el valor de 2 para comprobar la fuerza de la asociación.

\section{Resultados}

La Tabla 1 recoge los resultados de los análisis referidos a las competencias matemáticas básicas. El efecto principal de grupo resultó estadísticamente significativo [Wilk's Lambda $(\Lambda)=.697$, $\left.\mathrm{F}_{8,84}=4.56, p<.001, \eta^{2} p=.303\right]$. Los ANCOVA's de confirmación evidenciaron diferencias estadísticamente significativas en las habilidades de seriación $\left(\mathrm{F}_{1}, 91=5.34, p=.023, \eta^{2} p=.055\right)$, conservación $\left(\mathrm{F}_{1}, 81=9.04, p=.003, \eta^{2} p=.090\right)$, conteo procedimental $\left(\mathrm{F}_{1}, 81=14.35, p<.001\right.$, $\left.\eta^{2} p=.136\right)$, y comparación simbólica $\left(F_{1}, 81=21.05, p<.001, \eta^{2} p=.188\right)$. No se encontraron diferencias estadísticamente significativas para las competencias de clasificación $\left(F_{1}, 81=005, p>\right.$ $\left..05, \eta^{2} p=.000\right)$, inclusión $\left(F_{1}, 81=2.45, p>.05, \eta^{2}{ }_{p}=.026\right)$, conteo conceptual $\left(F_{1}, 81=.006\right.$, $\left.p>.05, \eta^{2} p=.000\right)$ y comparación no-simbólica $\left(F_{1}, 81=.008, p>.05, \eta^{2} p=.000\right)$. 


\section{HABILIDADES MATEMÁTICAS INICIALES Y DIFICULTADES MATEMÁTICAS PERSISTENTES}

Tabla 1.

Diferencias entre los grupos (T1): competencias matemáticas básicas

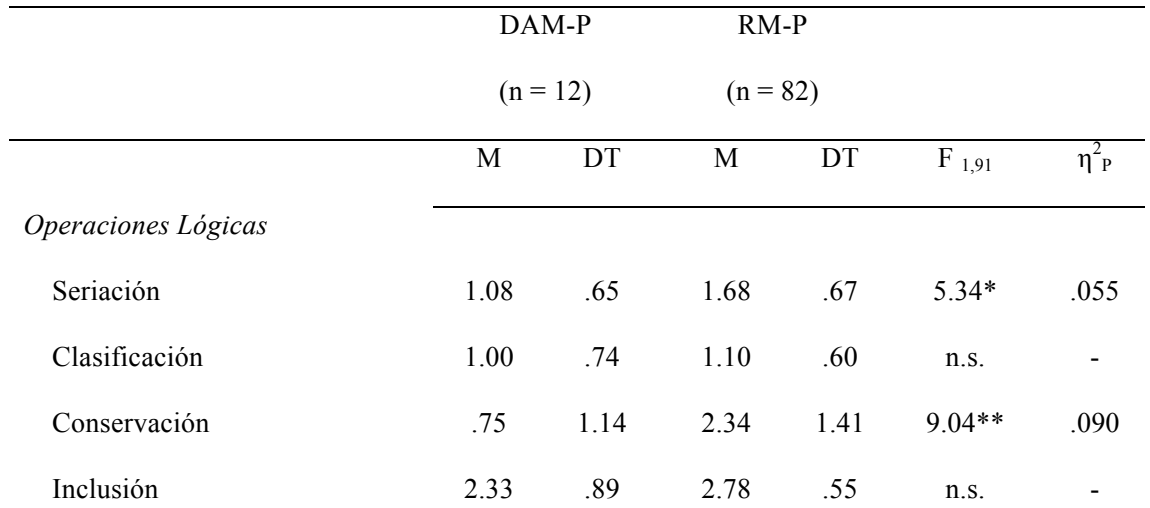

Conteo

$\begin{array}{lrrrrrr}\text { Procedimental } & 5.92 & 2.31 & 10.34 & 3.00 & 14.35^{* *} & .136 \\ \text { Conceptual } & 11.08 & .90 & 11.46 & 1.53 & \text { n.s. }\end{array}$

Numerosidad

Comparación simbólica

$\begin{array}{llllll}3.17 & .94 & 3.90 & .38 & 21.05 * * & .188\end{array}$

Comparación no-simbólica

$\begin{array}{lllll}5.92 & 69 & 5.89 & .29 & \text { n.s. }\end{array}$

Nota: DAM-P = Dificultades en el aprendizaje de las matemáticas persistentes; RM-P =

Rendimiento medio persistente

$* * p \leq .001 ; * p<.05 ;$ n.s. $=$ No significativo

\section{DISCUSIÓN}

El objetivo de esta investigación fue analizar las diferencias en las competencias matemáticas básicas evaluadas en El entre niños con dificultades persistentes y niños con rendimiento adecuado en el área de matemáticas dos años después, en $2^{\circ}$ de EP. Concretamente, las habilidades básicas que se analizan fueron conteo, operaciones lógicas y comparación de magnitudes.

Respecto a la competencia de conteo, los resultados mostraron una ejecución significativamente inferior del grupo con dificultades persistentes en tareas que requieren el manejo de la secuencia numérica verbal. Estos resultados están en concordancia con investigaciones previas realizadas con sujetos con dificultades específicas de aprendizaje de las matemáticas en los que se han identificado déficits en el conteo a nivel procedimental (Geary y Hoard, 2005; Jordan y Montani, 1997; Ostad, 2000). Sin embargo, no se encontraron diferencias en el conocimiento y uso de los principios conceptuales del conteo entre los grupos. Una posible explicación al respecto podría darse en base a que, tal y como informa Geary (2004), los sujetos con dificultades matemáticas no presentarían problemas en la comprensión de todos los conceptos que implican el conteo, tales 
como el orden estable y/o la cardinalidad, aspecto que podría hacer aumentar la puntuación global de conteo conceptual.

En el caso de las operaciones lógicas, se encontraron diferencias estadísticamente significativas entre los grupos con dificultades y rendimiento medio persistente en las tareas de seriación y conservación. En este sentido, los sujetos con dificultades persistentes presentarían dificultades en la comprensión del carácter inmutable de los conjuntos en base a criterios lógicos y, especialmente, en la organización de los elementos de un conjunto en función de sus diferencias, aspecto temprano cuya relevancia para el rendimiento matemático posterior ha sido previamente apuntada en diversos estudios longitudinales (Aunio y Niemivirta, 2010; Tobia, et al., 2015). Estos resultados son coincidentes con otros trabajos que han identificado los problemas ante tareas de operaciones lógicas como déficits característicos de aquellos sujetos que presentan dificultades matemáticas en etapas iniciales de la escolaridad (Stock, et al., 2009b; Stock, et al., 2010). No obstante, no se encontraron diferencias en las tareas de clasificación e inclusión, pese a la importancia que algunos estudios otorgan a estas habilidades para el rendimiento matemático (Stock, et al., 2009b). Este hecho podría relacionarse con el nivel de dificultad, la abstracción de la tarea y/o la etapa evolutiva en la que se encontraban los participantes, independientemente de la presencia de dificultades.

Finalmente, en la línea de los resultados obtenidos por Desoete et al. (2012), destaca especialmente el tamaño del efecto obtenido para las diferencias entre los grupos en la tarea de comparación de magnitudes cuando se utilizan números arábigos. Sin embargo, los resultados relativos a las tareas no-simbólicas no alcanzaron la significación estadística, en contraposición a otros trabajos (Bonny y Lourenco, 2013; Stock, et al, 2010). Cabe destacar que en dichos estudios se utilizaron sujetos de menor edad, así como niños con dificultades de diferente severidad, aspectos metodológicos que podrían justificar la ausencia de diferencias en la tarea no-simbólica en el presente estudio. Todo ello, parece indicar que uno de los déficits característicos de las dificultades matemáticas en los primeros cursos de EP tendría que ver con las dificultades en el acceso a la representación simbólica de las cantidades (Rousselle y Nöel, 2007).

Este trabajo no está exento de limitaciones. A pesar de tratarse de un estudio longitudinal, las evaluaciones se han realizado en dos momentos (El y $2^{0}$ curso de EP) únicamente. Sería muy interesante realizar más evaluaciones a lo largo de toda la escolaridad, ya que como se ha comentado anteriormente, los problemas en matemáticas tienen una continuidad en el tiempo (Mazzocco y Räsänen, 2013). Así mismo, son necesarios trabajos longitudinales que contemplen el peso explicativo que ofrecen otras variables de tipo personal (e.g. funcionamiento ejecutivo, motivacionales, ansiedad) como contextual (e.g. instruccionales, socio-ambientales, institucionales), con el objetivo de ofrecer una perspectiva comprensiva de los factores implicados en las dificultades matemáticas y en su persistencia. Además, futuros trabajos deberían incluir grupos de estudiantes con diferente grado de dificultad, con el objetivo de abarcar el continuo que implica las dificultades de aprendizaje en matemáticas.

Por último, del presente estudio se desprenden implicaciones para la práctica psicoeducativa. Sería interesante evaluar a nivel de screening, desde Educación Infantil, distintas habilidades matemáticas específicas como potenciales marcadores del rendimiento matemático posterior. La importancia de habilidades como las habilidades de comparación simbólica, el conteo procedimental, 0 las habilidades lógicas de seriación o conservación (aspecto al que, actualmente, se le destina menor atención) podrían ser aspectos importantes para la detección del riesgo de dificultades en el aprendizaje de las matemáticas y, consecuentemente, para la determinación de la persistencia de dichas dificultades. 


\section{HABILIDADES MATEMÁTICAS INICIALES Y DIFICULTADES MATEMÁTICAS PERSISTENTES}

\section{CONCLUSIONES}

Se puede concluir que los resultados muestran diferencias significativas en las habilidades matemáticas básicas entre los niños con y sin dificultades matemáticas persistentes. Concretamente, son las habilidades de conservación, conteo procedimental y comparación simbólica las que más diferencian a estos grupos.

Financiación: Este trabajo ha sido financiado por el Ministerio de Economía y Competitividad de España (EDU2012-37452) y la Universitat Jaume I de Castellón (beca pre-doctoral; 2I005-PREDOC/2013/34).

\section{REFERENCIAS BIBLIOGRÁFICAS}

Aunio, P., y Niemivirta, M. (2010). Predicting children's mathematical performance in grade one by early numeracy skills. Learning and Individual Differences, 20, 427-435.

Bonny, J. W., y Lourenco, S. F. (2013). The approximate number system and its relation to early math achievement: Evidence from the preschool years. Journal of Experimental Child Psychology, 114(3), 375-388.

Butterworth, B. (2005). Developmental dyscalculia. En Campbell, J. I. (Ed.) Handbook of mathematical cognition. Psychology Press.

Bynner, J. 2004. Literacy, numeracy and employability: evidence from the British birth cohort studies. Literacy and Numeracy Studies, 13(1), 1-48.

De Smedt, B., y Gilmore, C. K. (2011). Defective number module or impaired access? Numerical magnitude processing in first graders with mathematical difficulties. Journal of Experimental Child Psychology, 108(2), 278-292.

De Smedt, B., Noël, M. P., Gilmore, C., y Ansari, D. (2013). How do symbolic and non-symbolic numerical magnitude processing skills relate to individual differences in children's mathematical skills? A review of evidence from brain and behavior. Trends in Neuroscience and Education, 2(2), 48-55.

Desoete, A., Ceulemans, A., De Weerdt, F., y Pieters, S. (2012). Can we predict mathematical learning disabilities from symbolic and non-symbolic comparison tasks in kindergarten? Findings from a longitudinal study. British Journal of Educational Psychology, 82(1), 64-81.

Desoete, A., Stock, P., Schapens, A., Baeyens, D., y Roeyers, H. (2009). Classification, seriation, and counting in grades 1,2, and 3 as two-year longitudinal predictors for low achieving in numerical facility and arithmetical achievement? Journal of Psychoeducational Assessment, 27(3), 252-264.

Geary, D. C. (2004). Mathematics and learning disabilities. Journal of Learning Disabilities, 37(1), 415.

Geary, D. C. (2011). Consequences, characteristics, and causes of mathematical learning disabilities and persistent low achievement in mathematics. Journal of Developmental and Behavioral Pediatrics, 32(3), 250.

Geary, D. C., y Hoard, M. K. (2005). Learning disabilities in arithmetic and mathematics. En Campbell, J. K. (Ed.), Handbook of Mathematical Cognition (pp. 253-268). New York, NY: Taylor \& Francis.

Geary, D. C., Hoard, M. K., Nugent, L., y Bailey, D. H. (2013). Adolescents' functional numeracy is predicted by their school entry number system knowledge. PloS one, 8(1), e54651.

Ginsburg, H., y Baroody, A. (2003). TEMA-3; Test de Competencia Matemática Básica. Madrid: TEA.

Grégoire, J., Nöel, M., y Van Nieuwenhoven, C. (2005). TEDI-MATH; Test para el Diagnostico de las Competencias Básicas en Matemáticas. Madrid: TEA Ediciones. 
IBM Corp. Released 2013. IBM SPSS Statistics for Windows, Version 22.0. Armonk, NY: IBM Corp. IEA (2015). Estudio internacional de tendencias en matemáticas y ciencias. IEA. Informe Español. Jordan, N. C., y Montani, T. O. (1997). Cognitive Arithmetic and Problem Solving A Comparison of Children with Specific and General Mathematics Difficulties. Journal of Learning Disabilities, 30(6), 624-634.

Mazzocco, M. M., y Räsänen, P. (2013). Contributions of longitudinal studies to evolving definitions and knowledge of developmental dyscalculia. Trends in Neuroscience and Education, 2, 65-73.

Morgan, P. L., Farkas, G., y Wu, Q. (2009). Five-year growth trajectories of kindergarten children with learning difficulties in mathematics. Journal of Learning Disabilities, 42, 306-321.

Mullis, I. V. S., Martin, M. O., Foy, P., y Hooper, M. (2016). TIMSS 2015 International Results in Mathematics. Recuperado de: http://timssandpirls.bc.edu/ timss2015/ international-results/

Ostad, S. A. (2000). Cognitive subtraction in a developmental perspective: Accuracy, speed-of-processing and strategy-use differences in normal and mathematically disabled children. Focus on Learning Problems in Mathematics, 22(2), 18-32.

Östergren, R., y Träff, U. (2013). Early number knowledge and cognitive ability affect early arithmetic ability. Journal of Experimental Child Psychology, 115(3), 405-421.

Ping, R. M., y Goldin-Meadow, S. (2008). Hands in the air: Using ungrounded iconic gestures to teach children conservation of quantity. Developmental Psychology, 44, 1277-1287.

Rousselle, L., y Noël, M. P. (2007). Basic numerical skills in children with mathematics learning disabilities: A comparison of symbolic vs non-symbolic number magnitude processing. Cognition, 102(3), 361-395.

Schleifer, P., y Landerl, K. (2011). Subitizing and counting in typical and atypical development. Developmental Science, 14(2), 280-291.

Stock, P., Desoete, A., y Roeyers, H. (2010). Detecting children with arithmetic disabilities from kindergarten: evidence from a 3-year longitudinal study on the role of preparatory arithmetic abilities. Journal of Learning Disabilities, 4(3), 250-268.

Stock, P., Desoete, A., y Roeyers, H. (2009a). Screening for mathematical disabilities in kindergarten. Developmental Neurorehabilitation, 12, 389-396.

Stock, P., Desoete, A., y Roeyers, H. (2009b). Predicting arithmetic abilities: The role of preparatory arithmetic markers and intelligence. Journal of Psychoeducational Assessment, 27(3), 237251.

Tobia, V., Bonifacci, P., y Marzocchi, G. M. (2016). Concurrent and longitudinal predictors of calculation skills in preschoolers. European Journal of Psychology of Education, 31(2), 155-174. 
